\title{
Using Fractional-Order Differential Equations for Health Monitoring of a System of Cooperating Robots*
}

\author{
Kevin Leyden and Bill Goodwine ${ }^{1}$
}

\begin{abstract}
The dynamics of many large-scale robotic formation systems, including structured systems as well as some random scale-free networks of agents, can be accurately described using fractional-order differential equations. A fractional-order differential equation can contain derivative terms with noninteger order, e.g., the one-half derivative. This paper demonstrates that the fractional order of the dynamics of a system may be a potentially powerful new way to monitor the operational status of such systems. When the order of the system changes, it can indicate an important change in the status of the system. Integer-order models will never exhibit a change in order because the order is dictated by a natural first principle and the structure of the system. For this reason, traditional health monitoring tools essentially focus on identifying parameter variations in a mathematical description of the system, but not changes in order. When fractional-order models are considered, the infinite number of possible real-valued orders between any two integer orders may capture essential changes in the system's dynamics. This paper provides an example of such changes, provides a theoretical justification for the approach, and explores possible limitations to the approach.
\end{abstract}

\section{Motivational Example}

We start with the example that motivated this work. In the next section we provide a literature review and set the context for this work in the broader research area. In a previous ICRA paper we demonstrated that the following system is fractional-order in nature [1].

Consider a toxic waste spill and a system or robots deployed to map and/or clean up the spill. Generally in water or ground spills, the contaminant will diffuse and advect due to groundwater flow to the surrounding environment, as illustrated in Figure 1. In such a case, a system of robots deployed for remediation will need many robots where the contamination concentration is high and few where it is low. In the figure, the black circles represent the robots.

This task could be accomplished with a formation with a tree graph structure, an example of which is illustrated in Figure 2. In this case, there is one robot at the first generation, two at the second, four at the third, etc., where each successive generation with a greater number of robots is positioned in an area of greater contamination. To simplify the analysis, the robots in this example are constrained to move horizontally, and $x_{i j}$ represents the horizontal location of robot $j$ in the $i$ th generation. The robots maintain their relative positions by either a potential force represented by a spring with constant $k$ or by a damper-type relationship, $b$.

*The partial support of the US National Science Foundation under the CPS Large Grant No. CNS-1035655 is gratefully acknowledged.

${ }^{1}$ Both authors are with the Department of Aerospace \& Mechanical Engineering, University of Notre Dame, Notre Dame, IN 46556 USA, kleydenend.edu and billecontrols. ame.nd.edu

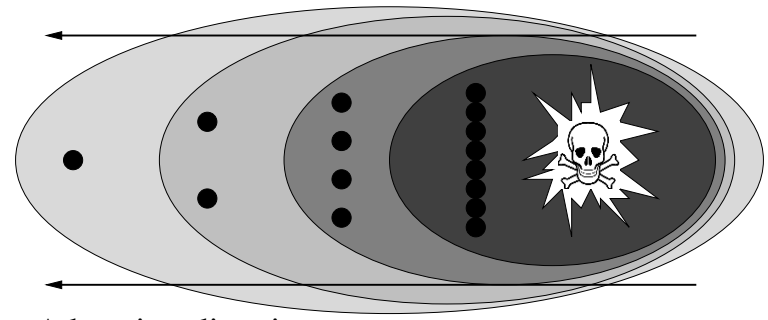

Advection direction

Fig. 1. Robotic environmental contamination remediation. Darker shaded regions indicate areas with greater contamination.

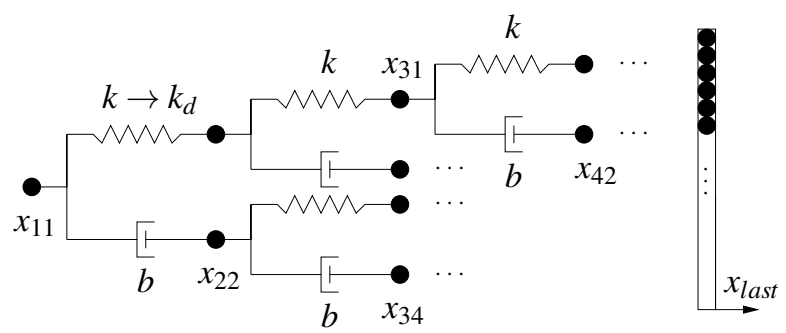

Fig. 2. Structure of robotic formation.

Figure 3 illustrates the frequency response of the relationship between the first robot, $x_{11}$, and the robots in the last generation, $x_{\text {last }}$, with a system of seven generations. The blue curve illustrates the case where all the spring constants are $k=2$, all the damper coefficients are $b=1$, the mass of the first robot is $m_{11}=1$, the mass of the robots in the seventh generation is 1 , and the masses of the interior robots are negligible.

The extended frequency range over which the phase is $-45^{\circ}$ and the slope of the magnitude curve is $-10 \mathrm{~dB} /$ decade suggests that the system may have $1 / 2$-order dynamics, and the results in [1] gave both analytical and numerical justifications for that interpretation. In Figure 3, the red curve is the frequency response for the case where some of the robots' interactions are degraded. Specifically, the system is "damaged" such that all parameters match the first case, except that one spring (the left-most one between $x_{11}$ and $\left.x_{12}\right)$ is reduced to $k_{d}=1 / 5$. The phase plot has shifted to $-60^{\circ}$, which suggests dynamics of fractional order $2 / 3$.

The rest of this paper supports this interpretation, both analytically and numerically. This suggests that changes in fractional order of a system could provide a novel and useful health monitoring tool for engineering systems, particularly robotic formation systems. 

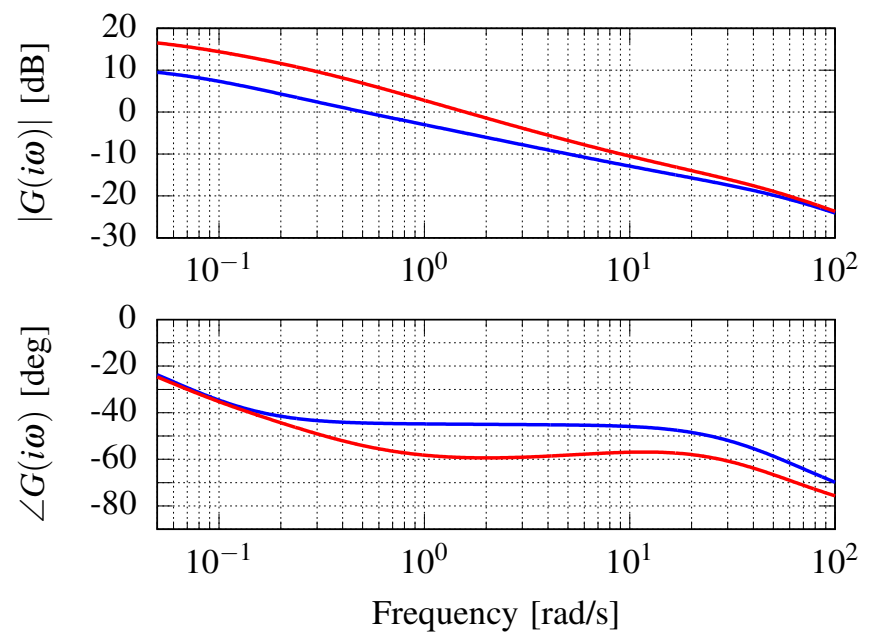

Fig. 3. Frequency response: blue curve undamaged, red curve damaged.

\section{BACKGROUND}

\section{A. Literature Review and Research Context}

Our work in [1], which considered the undamaged system in Figure 2, was motivated by a viscoelastic model from the literature [2], [3], and we showed that the system was accurately modeled by a differential equation with a halforder term. Because interacting, multi-agent systems are inherently of very high order, recognizing the fractionalorder dynamics of the system is important when a reducedorder and concise model is needed, and it will expand the set of tools available to roboticists when they need a model for design or analysis of their systems.

For the proposed system monitoring to be practical, it must be supported by a method of fractional-order system identification. Mathematical treatments of this topic include [4], which seeks a continuous order distribution to describe the system, since the order can be any real number. The method in [5] is motivated similarly but establishes a linear system of equations, yielding a discrete order distribution. A seriesbased approach to identifying governing equation parameters is detailed in [6]. Computational system identification procedures include the iterative optimization approach of [7]. Genetic algorithms are employed to optimize fractional-order governing equations in [8].

Multi-robot systems are well-studied and present interesting and challenging control problems. For example, see [9][14]. Some of the author's prior work is directed toward exact model reduction for symmetric systems [15]-[18]. Fractional calculus as a mathematical subject has a long history. Books on the mathematics and engineering applications include [19]-[21], and there are a number of review articles as well [22], [23]. One closely related study is [24], [25], concerning formation control of fractional systems. Those papers consider a problem in which the individual components are fractional in nature. In contrast, in this present paper, the fractional dynamics arise from the structure of the interaction among the agents. Other related studies include [26] (walking robots), [27], [28] (flexible manipulators), [29] (time delays) and control using fractional-order PID control [28], [30]. Our other prior papers on fractional calculus in engineering are [31], [32].

\section{B. Fractional Calculus}

It is natural to ask, given a function, $f(t)$, with a first derivative, $f^{(1)}(t)$, and second derivative, $f^{(2)}(t)$, whether there are operators "in between" the integer order derivatives such as

$$
\frac{d^{\frac{1}{2}}}{d t^{\frac{1}{2}}} f=f^{\left(\frac{1}{2}\right)}
$$

that generalize the notion of an integer-order derivative.

While closed-form solutions for fractional-order differential equations do exist (see our prior work for a brief summary or the other literature for more in-depth presentations), we also must resort to numerical approximations. To that end, consider the first and second derivatives of a function to be defined as

$$
\begin{aligned}
\frac{d f}{d t}(t) & =\lim _{\Delta t \rightarrow 0} \frac{f(t)-f(t-\Delta t)}{\Delta t} \\
\frac{d^{2} f}{d t^{2}}(t) & =\lim _{\Delta t \rightarrow 0} \frac{f(t)-2 f(t-\Delta t)+f(t-2 \Delta t)}{(\Delta t)^{2}}
\end{aligned}
$$

or in general for an integer $n$

$$
\frac{d^{n} f}{d t^{n}}(t)=\lim _{\Delta t \rightarrow 0} \frac{\sum_{0 \leq m \leq n}(-1)^{m}\left(\begin{array}{c}
n \\
m
\end{array}\right) f(t+(n-m) \Delta t)}{(\Delta t)^{n}},
$$

where the usual binomial coefficient is given by

$$
\left(\begin{array}{c}
n \\
m
\end{array}\right)=\frac{n !}{m !(n-m) !}
$$

This is easily generalized to non-integers by gamma functions

$$
\left(\begin{array}{c}
\alpha \\
m
\end{array}\right)=\frac{\Gamma(\alpha+1)}{\Gamma(m+1) \Gamma(\alpha-m+1)},
$$

giving the Grünwald - Letnikov derivative:

$$
\frac{d^{\alpha} f}{d t^{\alpha}}(t)=\lim _{\Delta t \rightarrow 0} \frac{1}{(\Delta t)^{\alpha}} \sum_{j=0}^{\infty}(-1)^{j}\left(\begin{array}{c}
\alpha \\
j
\end{array}\right) f(t+(\alpha-j) \Delta t) .
$$

If $\Delta t \ll 1$ and $t=m \Delta t$, then the time shift by $\alpha$ is small, and if all the initial conditions are zero, then we have

$$
\frac{d^{\alpha} f}{d t^{\alpha}}(t) \approx \frac{1}{(\Delta t)^{\alpha}} \sum_{j=0}^{m}(-1)^{j}\left(\begin{array}{c}
\alpha \\
j
\end{array}\right) f(t-j \Delta t),
$$

which is a useful approximation to solve fractional-order differential equations.

It is worth emphasizing that, unlike integer-order derivatives, fractional-order derivatives require more than local information. In fact, it is apparent from the sum in the definition in Equation 1, that all past values of a function enter into the computation for the fractional derivative.

\section{Mathematical ANALYsis}

This section presents a mathematical analysis relating the damaged and undamaged systems. 


\section{A. Undamaged System}

Observe that the tree network in Figure 2 is the composition of two types of transfer functions. The transfer function from the $x_{11}$ node to the $x_{21}$ node is $G_{1}(s)=1 / k$, and the transfer function from the $x_{11}$ node to the $x_{22}$ node is $G_{2}(s)=$ $1 /(b s)$. The entire system is parallel and series combinations of these. Specifically, if we consider the relationship between the total force created by relative displacements between the first and last generations, we can see that

$$
\frac{X_{11}(s)-X_{\text {last }}(s)}{F(s)}=\frac{1}{\frac{1}{G_{1}(s)+\frac{1}{G_{1}(s)+\frac{1}{G_{1}(s)+\cdots}}}+\frac{1}{G_{2}(s)+\frac{1}{G_{2}(s)+\frac{1}{G_{2}(s)+\cdots}}}}
$$

In the limit where there are an infinite number of generations, the transfer function from any node to the last generation is equal to the transfer function from the first node to the last generation; this is the mathematical manifestation of self-similarity in the network. If we denote that by $G_{\infty}(s)$, we have

$$
G_{\infty}(s)=\frac{1}{\frac{1}{G_{1}(s)+G_{\infty}(s)}+\frac{1}{G_{2}(s)+G_{\infty}(s)}}
$$

which can easily be solved for

$$
G_{\infty}(s)=\sqrt{G_{1}(s) G_{2}(s)} .
$$

This holds in general for any $G_{1}(s)$ and $G_{2}(s)$, and for the specific system in question we have

$$
G_{\infty}(s)=\frac{1}{\sqrt{k b s}}
$$

Because $s$ is the derivative operator in the frequency domain, the square root term in the denominator is indicative of $1 / 2$ order dynamics.

To further validate the 1/2-order dynamics, we use Equation 1 and numerically compute the response of the last generation, $x_{\text {last }}$, when $x_{11}$ is displaced. In particular, we take

$$
x_{11}= \begin{cases}t, & 0 \leq t<1 \\ 2-t, & 1 \leq t<2 \\ 0, & t \geq 2\end{cases}
$$

and Newton's law on the last generation with the transfer function in Equation 2 gives

$$
m s^{2} X_{\text {last }}(s)=\sqrt{k b s}\left(X_{1}(s)-X_{\text {last }}(s)\right)
$$

or in the time domain

$$
m \frac{d^{2} x_{\text {last }}}{d t^{2}}(t)+\sqrt{k b} \frac{d^{\frac{1}{2}} x_{\text {last }}}{d t^{\frac{1}{2}}}(t)=\sqrt{k b} \frac{d^{\frac{1}{2}} x_{1}}{d t^{\frac{1}{2}}}(t)
$$

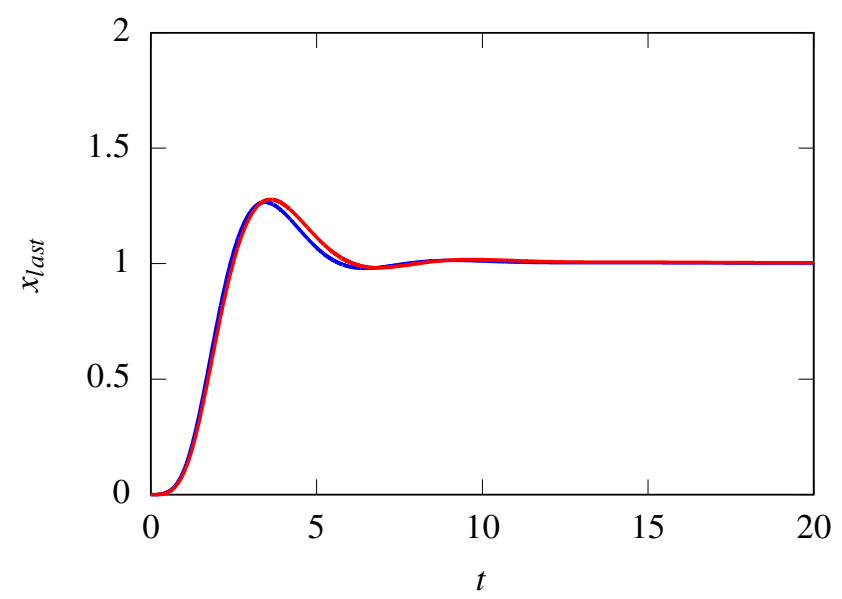

Fig. 4. Comparison of solution to Equation 3 to full dynamics of an eight-generation system. Red: fractional response, blue: full response.

Using Equation 1 and solving for $x_{\text {last }}$ at $t=n \Delta t$ gives

$$
\begin{aligned}
x_{\text {last }}(n \Delta t) & \approx\left(\frac{1}{\frac{m}{(\Delta t)^{2}}+\frac{\sqrt{k b}}{\sqrt{\Delta t}}}\right) \\
& \times\left[\frac{m}{(\Delta t)^{2}}(2 x((n-1) \Delta t)-x((n-2) \Delta t))\right. \\
& -\sum_{j=1}^{n}(-1)^{j}\left(\begin{array}{c}
\frac{1}{2} \\
j
\end{array}\right) x_{\text {last }}((n-j) \Delta t) \\
& \left.+\frac{\sqrt{k b}}{\sqrt{\Delta t}} \sum_{j=0}^{n}(-1)^{j}\left(\begin{array}{c}
\frac{1}{2} \\
j
\end{array}\right) x_{1}((n-j) \Delta t)\right] .
\end{aligned}
$$

Figure 4 compares the fractional-order response to the response of the full system with eight generations when $k=$ $2, b=1$, and $m=1$. The fractional-order approximation was a single fractional-order equation, and the full system had 255 second-order differential equations. Clearly the solutions are very close, and the fractional equation provides a very good approximation for the full system.

\section{B. Damaged System}

Consider the system where the leftmost spring has a different value that has been changed from its nominal value. In such a case, in the limit of an infinite number of generations with $k_{d}=k+\varepsilon$,

$$
G_{\text {damaged }}(s)=\frac{1}{\frac{1}{\frac{1}{k+\varepsilon}+\frac{1}{\sqrt{k b s}}}+\frac{1}{\frac{1}{b s}+\frac{1}{\sqrt{k b s}}} .}
$$

Note that this is not self-similar because of the perturbation on the first spring, but the generations greater than one are self-similar, indicated by the $1 / \sqrt{k b s}$ terms. Simplifying gives

$$
G_{\text {damaged }}(s)=\frac{(k+\varepsilon) \sqrt{b k s}+b s(\sqrt{b k s}+2 k+\varepsilon)}{b s\left(k(2 \sqrt{b k s}+b s+\varepsilon)+2 \varepsilon \sqrt{b k s}+k^{2}\right)} .
$$



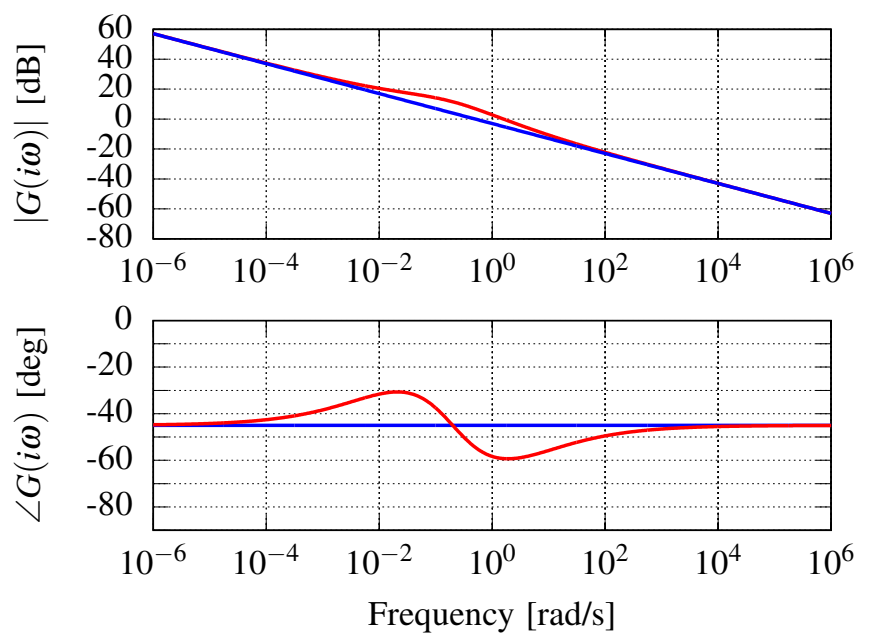

Fig. 5. Frequency response of damaged system with an infinite number of generations (red) compared to a half-order system frequency response (blue).
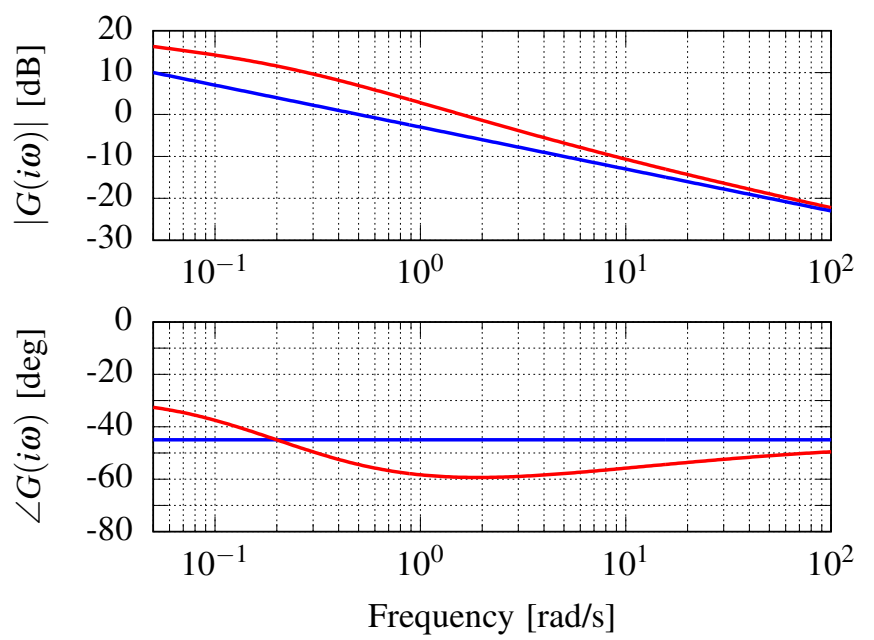

Fig. 6. Frequency response of damaged system with an infinite number of generations. Red: undamaged system with an infinite number of generations. Blue: damaged first generation followed by infinite number of undamaged generations.

The frequency response of this irrational transfer function is illustrated in Figure 5 with $k_{d}=1 / 5(\varepsilon=-9 k / 10), b=1$, and $m=1$.

Figure 6 is the same frequency response, but limited to be over the same frequency range as Figure 3. It clearly illustrates the same shift in frequency to approximately $-60^{\circ}$ at $\omega \approx 1$, which we saw in the original damaged system in Figure 3, corresponding to an order of approximately $2 / 3$. Furthermore the magnitude plot is shifted by approximately $7 \mathrm{~dB}$, which corresponds to a decrease in the force produced by the network by a factor of 0.45 .

Finally, we validate the response by computing the time domain response of a seven-generation system with the damaged spring and a fractional-order system with order 0.6. The full system will be the same as previously, but with

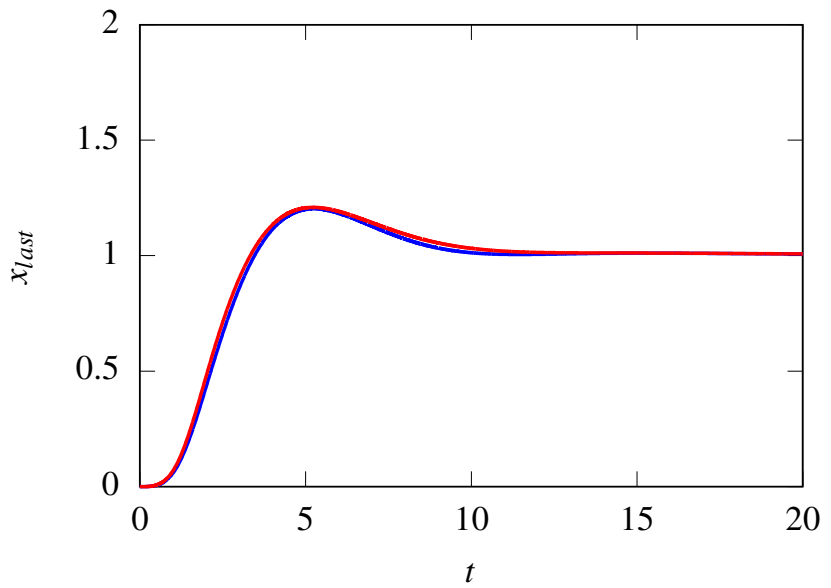

Fig. 7. Comparison of 2/3-order fractional solution (blue) to full solution (red) illustrating good agreement for damaged system.

$k_{d}=1 / 5$. The fractional system will be the solution to

$$
\begin{aligned}
x_{\text {last }}(n \Delta t) & \approx\left(\frac{1}{\frac{m}{(\Delta t)^{2}}+\frac{M \sqrt{k b}}{(\Delta t)^{0.6}}}\right) \\
& \times\left[\frac{m}{(\Delta t)^{2}}(2 x((n-1) \Delta t)-x((n-2) \Delta t))\right. \\
& -\sum_{j=1}^{n}(-1)^{j}\left(\begin{array}{c}
0.6 \\
j
\end{array}\right) x_{\text {last }}((n-j) \Delta t) \\
& \left.+\frac{M \sqrt{k b}}{(\Delta t)^{0.6}} \sum_{j=0}^{n}(-1)^{j}\left(\begin{array}{c}
\frac{1}{2} \\
j
\end{array}\right) x_{1}((n-j) \Delta t)\right],
\end{aligned}
$$

which is the same fractional solution as before, except with the order being 0.6 instead of $1 / 2$ and scaling the force, $\sqrt{k b}$, by a factor of $-7 \mathrm{~dB}$. A comparison of the solutions where $k=2, b=1, m=1$ is illustrated in Figure 7, which clearly indicates that the predicted change in order accurately represents the change in dynamics of the damaged system.

The implication of the result in Figure 7 is that the change in a parameter of the system, in this case $k \rightarrow k_{d}$, produces a predictable change in the fractional order of the system from $1 / 2$ to $2 / 3$. This, in turn, implies that if we are able to monitor or periodically check the order of a system, that may provide useful information regarding the status of the system.

\section{Another Example}

Consider the same system, but now decrease the coefficient of the leftmost damper from $b=1$ to $b=1 / 10$. The frequency response for this system is illustrated in Figure 8. Unlike the damaged spring, this shows a decrease in order from $1 / 2$ to approximately $1 / 3$. Like the damaged spring case, the magnitude term will be decreased, in this case by approximately $10^{-8 / 20}$. 

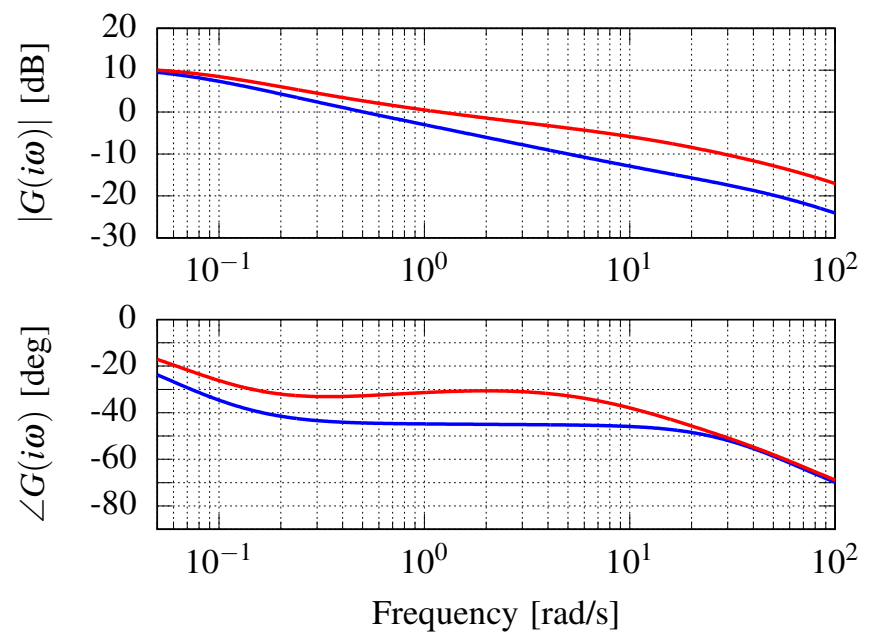

Fig. 8. Frequency response for damaged system with reduced damping in the first generation.

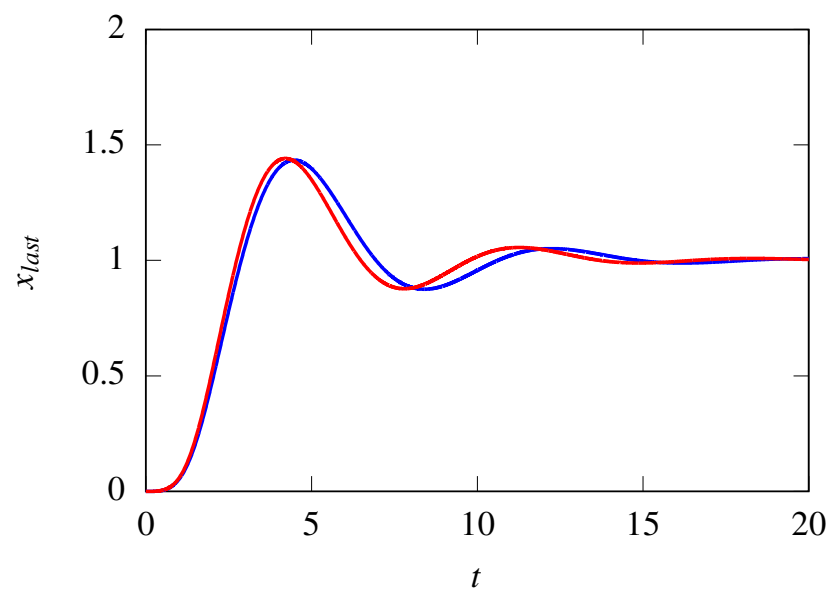

Fig. 9. Fractional vs exact response for damaged damper.

In this case the equation we solved numerically was

$$
\begin{aligned}
x_{\text {last }}(n \Delta t) & \approx\left(\frac{1}{\frac{m}{(\Delta t)^{2}}+\frac{M \sqrt{k b}}{(\Delta t)^{0.33}}}\right) \\
& \times\left[\frac{m}{(\Delta t)^{2}}(2 x((n-1) \Delta t)-x((n-2) \Delta t))\right. \\
& -\sum_{j=1}^{n}(-1)^{j}\left(\begin{array}{c}
0.33 \\
j
\end{array}\right) x_{\text {last }}((n-j) \Delta t) \\
& \left.+\frac{M \sqrt{k b}}{(\Delta t)^{0.33}} \sum_{j=0}^{n}(-1)^{j}\left(\begin{array}{c}
\frac{1}{2} \\
j
\end{array}\right) x_{1}((n-j) \Delta t)\right]
\end{aligned}
$$

with $M=10^{-8 / 20}$. Again, the fractional-order response shows close agreement with the full dynamics.

\section{DISCUSSION}

The following are findings from preliminary investigations into other damage cases for this example system. These inform the potential scope of fractional order measurement as a monitoring tool.

\section{A. Damage Detection}

The previous sections consider damage to the leftmost spring and damper. In the example system, the springs and dampers are assumed identical before damage, so the effects of damage to the other components in the formation are of interest as well. The components that have the greatest effect on the system's frequency response are those in serial connection with only like elements. These comprise the upper (all springs) and lower (all dampers) edges of the tree diagram in Figure 2 and will be referenced as the critical components.

While the damage to the leftmost spring and damper appear to bring about a change in order, damage to the other critical components brings about different behaviors in the frequency response. The extent of these frequency response adjustments is dependent on which component is damaged. Naturally, damages on the left side of the formation tree are more severe because those components are connected to significant portions of the tree. Indeed, there is a progression in the appearance of the frequency response plots from the cases of most critical damage to that of no damage.

This progression relates to the concavity of the phase difference. Damage to critical springs yields a similar response to the damage case in Figure 3, except that for high frequencies, the behavior more closely resembles the undamaged system. Therefore, the progression is described as follows: there is a frequency band over which the effect of the damage is seen. The deeper in the formation the damage occurs, and thus the less critical the damage, the smaller that frequency band.

If the damaged element is a damper, the result has characteristics opposite to the spring damage cases. The system response mimics the undamaged system response for lower frequencies and the case of worst damage for higher frequencies. The apparent duality between the results from the spring and damper cases despite clear dynamical differences between those elements could perhaps be exploited in an extension of this method.

\section{B. Limitations}

Thus far, this discussion has neglected the springs and dampers that are not on the outer edges of the tree diagram in Figure 2. Damaging these inner components has negligible effect on the overall frequency response. The damage causes some change in the full dynamics of the system, but there are no emergent properties in these new dynamics that differ in any meaningful sense from the undamaged system.

Compounding of these subtle damages might eventually cause more severe dynamical changes. In an extreme case where each of the inner element coefficients, $k$ or $b$, is reduced to $1 / 10$ of its normal operating value, the frequency band suggestive of fractional-order dynamics vanishes. This aligns with intuition, since that level of damage would essentially reduce the system to two elements: a series of 
springs with an effective stiffness constant and a series of dampers with an effective damping constant.

A different type of compounding that could hinder damage detection is one in which $k$ and $b$ may both change for a spring-damper pair, but their product is approximately preserved. This is because $k$ and $b$ are multiplied in Equation 2 and, in turn, the governing equation. It is unclear what type of damage might have this effect. Our current efforts are directed toward defining the extent of these limitations, or correspondingly, the limits of the efficacy of our proposed approach.

\section{Implementation}

The application of this method to a physical system would first require frequency data from an operational state. Similar data would then be collected from the damaged state. If the order of dynamic response evident in the medium-frequency range is higher than expected, the damage would likely have occurred in an elastic component; if lower, a damping component. Furthermore, the location of the damaged element could be estimated by measuring the frequency band in which the response mimics that of a damaged system. The wider this band is, the more critical the location of the damage. The full extent of applicability for this method is not yet known.

\section{CONCLUding REMARKS}

This paper has presented a new method for detection of damage to a component in a system such as a robotic formation. The damage may be seen as a result of a change in the fractional order of the system's governing equation. Given the ubiquity of models based on spring and damper elements, this method has the potential to be widely useful in structural or systemic health monitoring.

Three main directions of research to build upon these preliminary findings are apparent. The first looks inward; the method should be shown to deliver similar results for other example systems. These results would include more specific quantitative findings; however, every physical system is different, so the main advantage is the transparent nature of the differences between frequency responses.

The second is pursuit of further consolidation of this method with the theory of fractional calculus and differential equations. For instance, there may be an explicit relationship between the governing equation of a damaged system and the equation for a fully operational one, given a specific type and extent of damage. Additional mathematical analysis would solidify the intuitively affirmative results thus far.

The third is extension into the realms of system identification and structural health monitoring, which is indeed the primary motivation for these advances. The former suggests issues of robustness to be examined; the latter, fundamental questions about the utility of the method for other types of models. These three directions point to a versatile approach for detecting damage in a system in light of the fractionalorder behavior evident in its frequency response.

\section{REFERENCES}

[1] Bill Goodwine. Modeling a multi-robot system with fractional-order differential equations. In Proceedings of the IEEE International Conference on Robotics and Automation, pages 1763-1768, 2014.

[2] N. Heymans and J.C. Bauwens. Fractal rheological models and fractional differential equations for viscoelastic behavior. Rheologica Acta, 33:210219, 1994.

[3] Jayson Mayes. Reduction and Approximation in Large and Infinite Potential-Driven Flow Networks. PhD thesis, University of Notre Dame, 2012.

[4] Tom T. Hartley and Carl F. Lorenzo. Fractional-order system identification based on continuous order-distributions. Signal processing, 83(11):2287-2300, 2003.

[5] Shantanu Das. Functional fractional calculus. Springer Science \& Business Media, 2011.

[6] Da-Yan Liu, Taous-Meriem Laleg-Kirati, Olivier Gibaru, and Wilfrid Perruquetti. Identification of fractional order systems using modulating functions method. In American Control Conference (ACC), 2013, pages 1679-1684. IEEE, 2013.

[7] Arun Narang, Sirish L. Shah, and T. Chen. Continuous-time model identification of fractional-order models with time delays. IET Control Theory \& Applications, 5(7):900-912, 2011.

[8] Shengxi Zhou, Junyi Cao, and Yangquan Chen. Genetic algorithmbased identification of fractional-order systems. Entropy, 15(5):16241642, 2013.

[9] Wei Ren, Randal W. Beard, and Ella M. Atkins. Information consensus in multivehicle cooperative control. IEEE Control Systems Magazine, pages 71-82, April 2007.

[10] J. Alexander Fax and Richard M. Murray. Information flow and cooperative control of vehicle formations. IEEE Transactions on Automatic Control, 49(9):1465- 1476, 2004.

[11] N.E. Leonard and E. Fiorelli. Virtual leaders, artificial potentials, and coordinated control of groups. In Proceedings of the 40th IEEE Conference on Decision and Control, pages 2968-2973, December 2001.

[12] Aveek K. Das, Rafael Fierro, Vijay Kumar, James P. Ostrowski, John Spletzer, and Camillo J. Taylor. A vision-based formation control framework. IEEE Transactions on Robotics and Automation, 18(5):813-825, 2002.

[13] Richard M Murray. Recent research in cooperative control of multivehicle systems. Journal of Dynamic Systems Measurement and Control, 129(5):571, 2007.

[14] Yongcan Cao, Wenwu Yu, Wei Ren, and Guanrong Chen. An overview of recent progress in the study of distributed multi-agent coordination. Industrial Informatics, IEEE Transactions on, 9(1):427-438, 2013.

[15] M. Brett McMickell and Bill Goodwine. Reduction and non-linear controllability of symmetric distributed systems. International Journal of Control, 76(18):1809-1822, 2003.

[16] M. Brett McMickell and Bill Goodwine. Motion planning for nonlinear symmetric distributed robotic formations. The International journal of robotics research, 26(10):1025-1041, 2007.

[17] Bill Goodwine and Panos J. Antsaklis. Multi-agent compositional stability exploiting system symmetries. Automatica, pages 3158-3166, 2013.

[18] M. Brett McMickell and Bill Goodwine. Reduction and non-linear controllability of symmetric distributed systems. In Proceedings of the IEEE International Conference on Robotics and Automation, pages 3454-3460, 2002.

[19] Keith B. Oldham and Jerome Spanier. The Fractional Calculus. Academic Press, New York, 1974.

[20] Dumitru Baleanu, Jos Antnio Tenreiro Machado, and Albert C. J. Luo. Fractional Dynamics and Control. Springer Publishing Company, Incorporated, 2011.

[21] Manuel Duarte Ortigueira. Fractional Calculus for Scientists and Engineers, volume 84 of Lecture Notes in Electrical Engineering. Springer, 2011.

[22] M.D. Ortigueira. An introduction to the fractional continuous-time linear systems: the 21st century systems. Circuits and Systems Magazine, IEEE, 8(3):19-26, 2008.

[23] J. Tenreiro Machado, Virginia Kiryakova, and Francesco Mainardi. Recent history of fractional calculus. Communications in Nonlinear Science and Numerical Simulation, 16(3):1140 - 1153, 2011.

[24] Yongcan Cao and Wei Ren. Distributed formation control for fractional-order systems: Dynamic interaction and absolute/relative damping. Systems \& Control Letters, 59(34):233 - 240, 2010. 
[25] Yongcan Cao, Yan Li, Wei Ren, and Yang Quan Chen. Distributed coordination of networked fractional-order systems. Systems, Man, and Cybernetics, Part B: Cybernetics, IEEE Transactions on, 40(2):362$370,2010$.

[26] Manuel F Silva, JA Tenreiro Machado, and AM Lopes. Fractional order control of a hexapod robot. Nonlinear Dynamics, 38(1-4):417433, 2004.

[27] H. Delavari, P. Lanusse, and J. Sabatier. Fractional order controller design for a flexible link manipulator robot. Asian Journal of Control, 15:783795, 2013.

[28] Chunna Zhao, Dingyu Xue, and YangQuan Chen. A fractional order pid tuning algorithm for a class of fractional order plants. In Proceedings of the IEEE International Conference on Mechatronics \& Automation, 2005.

[29] YangQuan Chen and Kevin L. Moore. Analytical stability bound for a class of delayed fractional-order dynamic systems. Nonlinear Dynamics, 29(1-4):191-200, 2002.

[30] Concepcin A. Monje, Blas M. Vinagre, Vicente Feliu, and YangQuan Chen. Tuning and auto-tuning of fractional order controllers for industry applications. Control Engineering Practice, 16(7):798 - 812, 2008.

[31] Bill Goodwine. Fractional-order dynamics in a random, approximately scale-free network of agents. In Proceedings of the IEEE Conference on Control Automation Robotics \& Vision, pages 1581-1586, 2014.

[32] Bill Goodwine and Kevin Leyden. Recent results in fractionalorder modeling for multi-agent systems and linear friction welding. Extended abstract. 